\title{
CONNECTIVITY, DIVISIBILITY, AND TORSION $\left({ }^{1}\right)$
}

\author{
BY \\ LEWIS C. ROBERTSON
}

Introduction. All of the groups discussed below are locally compact abelian groups, and additive notation is employed whenever convenient. A list at the end of the paper provides references to the pertinent definitions and notations.

In $\S 1$ we define $Z$-groups by a constructive process. The basic idea is that of starting with the integers and the finite cyclic groups, and taking all groups which can be obtained by repeatedly including dual groups, including minimal divisible extensions, and including local direct products. $\$ 1$ continues with some of the easier results on $Z$-groups, several of which are listed below.

$\$ 2$ contains a discussion of certain important $Z$-groups, and some counterexamples.

$\$ 3$ discusses properties related to connectivity, divisibility, and torsion, and shows the significance which $Z$-groups have in the analysis of these properties. A characterization is obtained of the dual of a connected group.

$\$ 4$ gives a complete classification of homogeneous groups, and some results on endomorphism-simple groups.

In $\$ 5$ we conclude with some applications of the classification of homogeneous groups. These include a characterization of the dual of a torsion-free group, a classification of certain nonabelian automorphism groups, and results on the structure of division rings.

Since $Z$-groups continue to appear throughout the various sections, we summarize the main results on $Z$-groups. Let $H$ be a locally compact abelian group which has compact identity component. Then $H$ is a $Z$-group if any of the following conditions are satisfied:

$H$ discrete and finite, finitely generated, divisible, or free.

$H$ torsion-free and compact or divisible.

$H$ endomorphism-simple and a $p$-group.

$H$ the additive group of a locally compact field.

$H$ homogeneous.

Moreover, every connected $H$ is a quotient group of a $Z$-group, every $H$ which is torsion-free or endomorphism-simple is an open subgroup of a $Z$-group, and every $H$ is a direct limit of groups which are inverse limits of $Z$-groups. The results on

Received by the editors September 2, 1965.

(') This material is part of the author's Ph.D. thesis submitted to the University of California, Los Angeles, January, 1965.

The research was in part financed by National Science Foundation Grant GP-2045. 
groups with compact identity component are useful, since an arbitrary locally compact abelian $G$ can be decomposed as $G=R^{n}+H$, where $H$ has compact identity component $[4$, p. 389].

ACKNOWLEDGEMENTS. I am extremely indebted to my thesis advisor, Professor Robert J. Blattner, whose help and encouragement have been invaluable. Several other individuals played various parts in shaping the form and content of this paper, among them R. Arens, A. Figá-Talamanca, S. Franklin, K. V. R. Rao, M. Rajagopalan, and H. G. Senge.

1. The category of $Z$-groups. Let $(G, K(G))$ be a pair of groups such that $K(G)$ is a compact open subgroup of $G$, and let $\left(\hat{G}, K(G)^{\perp}\right)$ denote the pair consisting of $\hat{G}$, the character group of $G$, and $K(G)^{\perp}$, the annihilator in $\hat{G}$ of $K(G)$. Note that $K(G)^{\perp}$ is a compact open subgroup of $\hat{G}$, since the annihilator of a compact subgroup is open, and the annihilator of an open subgroup is compact. Let $E(G)$ denote the minimal divisible extension of $G[4$, p. 419 and p. 445]. Then $(E(G), K(G))$ is a pair of groups such that $K(G)$ is an open subgroup of $E(G)$, since $G$ is by definition an open subgroup of $E(G)$.

Let $S$ be a set of pairs $(G, K(G))$ such that, for each $G, K(G)$ is a uniquely defined compact open subgroup of $G$. If $\mu$ is an ordinal number, we say that $M\left(\mu, G_{x}\right)$ is a direct product of $S$-groups over $\mu$ if there is a function $\alpha: \mu \rightarrow S$ such that $\left(G_{x}, K\left(G_{x}\right)\right)=\alpha(x)$ for every $x \in \mu$, and $M\left(\mu, G_{x}\right)$ consists of all mappings $f$ defined on $\mu$ such that $f(x) \in G_{x}$ for every $x \in \mu$. Let $M\left(\mu, G_{x}\right)$ be a direct product of $S$ groups over $\mu$, and let $L\left(\mu, G_{x}, K\left(G_{x}\right)\right)$ denote the local direct product of the groups $G_{x}$ relative to the compact open subgroups $K\left(G_{x}\right)$. (See [2, p. 7], and also [4, p. 56].) $L\left(\mu, G_{x}, K\left(G_{x}\right)\right)$ is the subgroup of $M\left(\mu, G_{x}\right)$ consisting of those $f \in M\left(\mu, G_{x}\right)$ such that $f(x) \in K\left(G_{x}\right)$ for all but a finite number of $x \in \mu$. Note that $M\left(\mu, K\left(G_{x}\right)\right)$ is by definition a compact open subgroup of $L\left(\mu, G_{x}, K\left(G_{x}\right)\right)$. We say that $L\left(\mu, G_{x}, K\left(G_{x}\right)\right)$ is a local direct product of $S$-groups over $\mu$. If $G_{x}=G$ for all $x \in \mu$, we delete the subscript $x$ and write $M(\mu, G)$. Note that a strong direct product $M(\mu, G)$ is just a local direct product of the form $L(\mu, G, G)$, whereas a weak direct product is just a local direct product of the form $L(\mu, G, e)$.

We now inductively define the class of $Z$-pairs. Let $S_{1}$ denote the set of pairs $(G, e)$, where $G$ is a cyclic group (perhaps with only one element), and $e$ is the identity subgroup. $(G, e) \in S_{1}$ if and only if $G$ is a homomorphic image of the integers $Z$. Given an ordinal number $\mu$, suppose that the set $S_{\mu}$ has been defined. Let $\tilde{S}_{\mu}=A_{\mu} \cup B_{\mu} \cup C_{\mu}$, where $A_{\mu}=S_{\mu}, B_{\mu}$ is the set of all $\left(\hat{G}, K(G)^{\perp}\right)$ such that $(G, K(G)) \in S_{\mu}$, and $C_{\mu}$ is the set of all $(E(G), K(G))$ such that $(G, K(G)) \in S_{\mu}$. Define $S_{\mu+1}$ as the set of all pairs $\left(L\left(\mu+1, G_{x}, K\left(G_{x}\right)\right), M\left(\mu+1, K\left(G_{x}\right)\right)\right)$ such that $L\left(\mu+1, G_{x}, K\left(G_{x}\right)\right)$ is a local direct product of $\tilde{S}_{\mu}$-groups over $\mu+1$. If $\lambda$ is a limit ordinal, define the set $S_{\lambda}$ as the union of the sets $S_{\mu}, \mu<\lambda$. $(G, K(G))$ is by definition a $Z$-pair if and only if $(G, K(G)) \in S_{\mu}$ for some $\mu$. Having defined the class of $Z$-pairs, we say that $G$ is a $Z$-group if and only if $G$ is the left-hand member of a $Z$-pair $(G, K(G))$. 
1.1. Proposition. Every Z-group has compact identity component.

Proof. Every $Z$-group has a compact open subgroup.

1.2. Proposition. Every finitely generated discrete abelian group is a Z-group.

Proof. Every finitely generated abelian group is a direct product of $S_{1}$-groups over some finite cardinal, and a finite product of $Z$-groups is a $Z$-group.

1.3. Proposition. Every free abelian discrete group is a Z-group.

Proof. Every free abelian group is a local direct product $L(\mu, Z, e)$, where $e$ is the identity of the integers $Z$.

1.4. Proposition. Suppose the identity component of $G$ is compact. Then $G$ is a direct limit of groups which are inverse limits of Z-groups.

Proof. $G$ is a direct limit of its compactly generated open subgroups $H_{\alpha}$, by [10, p. 107]. Each $H_{\alpha}$ has compact open subgroups, since $H_{\alpha}$ is open in $G$, and $G$ has compact open subgroups. Each $H_{\alpha}$ is an inverse limit of groups of the form $R^{n}+T^{m}+A$, where $A$ is a finitely generated discrete group, by [10, p. 98]. The factors $R^{n}$ cannot appear, because quotient-group surjections are continuous open mappings, and $H_{\alpha}$ has compact open subgroups. Thus $H_{\alpha}$ is an inverse limit of groups of the form $T^{m}+A$. Each of the groups of the form $T^{m}+A$ is a $Z$-group, since the dual of a $Z$-group is a $Z$-group, and finite sums of $Z$-groups are $Z$-groups.

\subsection{Proposition. Every divisible discrete group is a Z-group.}

Proof. Every divisible discrete group is a local direct product $L\left(\mu, G_{x}, e\right)$, where each $G_{x}$ is either the discrete rationals $Q_{d}$ or else is $Z_{p}^{\infty}$ for some prime $p$ [5, p. 10]. Thus all the groups involved in the local direct product are $Z$-groups, since $Q_{d}$ is the minimal divisible extension of $Z$, and $Z_{p}^{\infty}$ is the minimal divisible extension of the $p$-element cyclic group $Z_{p}$.

COROLLARY 1.6. Every compact torsion-free group is a Z-group.

Proof. Duals of $Z$-groups are $Z$-groups, and we see by [4, Theorem 25.8 , p. 406] that $G$ is compact torsion-free if and only if $\hat{G}$ is discrete divisible.

1.7. Proposition. Every compact connected $G$ is a quotient group of a Z-group.

Proof. If $G$ is compact and connected, then $\hat{G}$ is torsion-free and discrete, by [4, Theorem 24.25, p. 385]. The injection from $\hat{G}$ to its minimal divisible extension $E(\hat{G})$ is a mapping $j$ from $\hat{G}$ to a torsion-free divisible discrete group [4, Theorem A.16, p. 446]. The transpose $j^{t}$ of $j$ is a quotient-group surjection from a compact group $A$ onto $G$, and $A$ is a $Z$-group, since $\hat{A}$ is the $Z$-group $E(\hat{G})$.

REMARK. If $G$ is compactly generated, then $G$ is connected if and only if $G$ is divisible. This follows from the decomposition $G=R^{n}+Z^{m}+K$, where $K$ is compact, by making use of the fact that a compact group $K$ is connected if and only if 
it is divisible. (See [4, Theorem 9.8, p. 90, and Theorem 24.25, p. 385].) The above Proposition 1.7 can be used as an alternative proof of the equivalence of connectivity and divisibility for compact groups; we need only show that the $Z$-group $A$ is connected and divisible. We shall frequently employ the device of establishing a result for certain particular $Z$-groups, and then generalizing to quotient groups.

\section{Some important $Z$-groups.}

2.1. Definitions and notations. $T$ denotes the circle, and $Z_{m}$ denotes the $m$-element cyclic group, regarded as a discrete subgroup of the circle. Given a prime number $p$, the minimal divisible extension $Z_{p}^{\infty}$ of $Z_{p}$ is the discrete group consisting of the union of all the cyclic groups $Z_{p^{n}}$. Given a cardinal number $\mu$, let $Z_{p}^{\infty}(\mu)$ denote the weak direct sum $L\left(\mu, Z_{p}^{\infty}, e\right)$ of $Z_{p}^{\infty}$ over the cardinal number $\mu$. Let $J_{p}$ denote the dual of $Z_{p}^{\infty}$. Then $J_{p}$ is the additive group of the ring of $p$-adic integers, and the minimal divisible extension $F_{p}$ of $J_{p}$ is the additive group of the $p$-adic number field which is obtained by completing the rationals relative to the metric of the $p$-adic valuation. (See [6, sections 19.12, 45.7, 50.4], and also [4, section 25.1].) Given a cardinal number $\mu, M\left(\mu, J_{p}\right)$ denotes the strong direct product $L\left(\mu, J_{p}, J_{p}\right)$. Let $M_{b}\left(\mu, F_{p}\right)$ denote the minimal divisible extension of $M\left(\mu, J_{p}\right)$. Then $M_{b}\left(\mu, F_{p}\right)$ is a $Z$-group, since we started with the cyclic group $Z_{p}$, and proceeded by minimal divisible extensions, dual groups, and local direct products.

2.2. Proposition. $M_{b}\left(\mu, F_{p}\right)$ consists of all bounded mappings from $\mu$ to $F_{p}$, that is, all $f: \mu \rightarrow F_{p}$ such that $|f(x)| \leqq B_{f}$ for every $x \in \mu$.

Proof. $F_{p}$ is divisible by definition, and it follows that the set of all mappings $M\left(\mu, F_{p}\right)$ from $\mu$ to $F_{p}$ is a divisible group which contains $M\left(\mu, J_{p}\right)$. Let $A$ be the subgroup of $M\left(\mu, F_{p}\right)$ consisting of all $p^{-n} f$ such that $f \in M\left(\mu, J_{p}\right)$. Let $k \in Z$ be an integer such that $k=p^{n} q$ with $q$ relatively prime to $p$. Then $k^{-1} A=A$, since $k$ is a unit in $J_{p}$. Thus $A$ is divisible, and consequently coincides with $M_{b}\left(\mu, F_{p}\right)$, since it is clear that no proper divisible subgroup of $A$ contains $M\left(\mu, J_{p}\right)$. It now follows readily that $M_{b}\left(\mu, F_{p}\right)$ is the group of all bounded mappings from $\mu$ to $F_{p}$, since $\|g\|_{\infty}=p^{n}$ if and only if $g=p^{-n} f$ with $\|f\|_{\infty}=1$.

2.3. Proposition. The quotient group of $M_{b}\left(\mu, F_{p}\right)$ with respect to the compact open subgroup $M\left(\mu, J_{p}\right)$ is a discrete divisible p-group $Z_{p}^{\infty}(\lambda)$. If $\mu$ is finite, then $\lambda=\mu$, whereas $\lambda \geqq 2^{\mu}$ if $\mu$ is infinite.

Proof. Let $D$ denote the quotient group in question. $D$ is discrete, since $M\left(\mu, J_{p}\right)$ is open by definition of $M_{b}\left(\mu, F_{p}\right) . D$ is divisible since it is a homomorphic image of the divisible group $M_{b}\left(\mu, F_{p}\right)$, and the above Proposition 2.2 shows that every $a \in D$ has order $p^{n(a)}$. Thus there is a cardinal number $\lambda$ such that $D=Z_{p}^{\infty}(\lambda)$, and this cardinal is unique [5, remark (b), p. 11]. If $\mu$ is finite, then $\lambda=\mu$ follows from the fact that $F_{p} / J_{p}=Z_{p}^{\infty}$. If $\mu$ is infinite, we consider the elements $p^{-1} f_{S}$ $\in M\left(\mu, F_{p}\right)$, where $f_{S}$ is the characteristic function of a subset $S$ of $\mu$. These elements 
are all distinct modulo $M\left(\mu, J_{p}\right)$, and there are $2^{\mu}$ of them. Thus the quotient group $Z_{p}^{\infty}(\lambda)$ has at least $2^{\mu}$ elements. On the other hand, $Z_{p}^{\infty}(\lambda)$ has $\lambda=\lambda \omega$ elements, since $Z_{p}^{\infty}$ has $\omega$ elements. Thus $\lambda \geqq 2^{\mu}$.

2.4. Counterexample. A continuous bijective automorphism of a locally compact abelian group $G$ need not be bicontinuous, even if this automorphism is multiplication by $p$. Take $G=M_{b}\left(\mu, F_{p}\right)$ with $\mu$ infinite. Multiplication by $p$ is always continuous, since addition is continuous. Multiplication by $p$ is surjective, since $M_{b}\left(\mu, F_{p}\right)$ is divisible, and is injective, since $M_{b}\left(\mu, F_{p}\right)$ is torsion-free. Thus multiplication by $p$ is a continuous bijective automorphism. Multiplication by $p^{-1}$ sends the compact group $M\left(\mu, J_{p}\right)$ onto a group $A$. $A$ is characterized by noting that the quotient group $A \bmod M\left(\mu, J_{p}\right)$ consists precisely of those elements of $Z_{p}^{\infty}(\lambda)$ which have order $p$. Thus $A$ cannot be compact, since it has an infinite discrete quotient group. This shows that multiplication by $p^{-1}$ is not continuous.

2.5. Proposition. $M_{b}\left(\mu, F_{p}\right)$ is torsion-free, and is the minimal divisible extension of any of its open subgroups.

Proof. $M_{b}\left(\mu, F_{p}\right)$ is torsion-free because $F_{p}$ is torsion-free. Proposition 2.3 shows that the quotient group $M_{b}\left(\mu, F_{p}\right) \bmod M\left(\mu, J_{p}\right)$ is a discrete $p$-group. Using the fact that $p^{n} t \rightarrow 0$ for any $t \in J_{p}$, it follows that $p^{n} f \rightarrow 0$ for any $f \in M\left(\mu, J_{p}\right)$ since $\left\|p^{n} f\right\|_{\infty}=p^{-n}\|f\|_{\infty}$. Hence $p^{n} g \rightarrow 0$ for any $g \in M_{b}\left(\mu, F_{p}\right)$, and so any discrete quotient group of $M_{b}\left(\mu, F_{p}\right)$ is a $p$-group. It is shown in [4, Theorem A.17, p. 446] that a torsion-free divisible group is the minimal divisible extension of any subgroup which yields a torsion group quotient.

2.6. Counterexample. The dual of a torsion-free group $G$ need not be divisible, even if $G$ is divisible. Taking $G=X$ in the example of [4, section 24.44(a), p. 393] shows that the dual of a torsion-free group need not be divisible. Here the group $X$ fails to be divisible, whereas Proposition 2.7 below gives a somewhat more natural example with $G$ divisible. If either $G$ or $\hat{G}$ is compactly generated, then $G$ is torsion-free if and only if $\hat{G}$ is divisible.

2.7. Proposition. The dual of $M_{b}\left(\mu, F_{p}\right)$ is divisible if and only if $\mu$ is finite.

Proof. Suppose that $\mu$ is finite, $\mu=n$. Then $M_{b}\left(\mu, F_{p}\right)$ is just $F_{p}^{n}$, since every mapping from $\mu$ is bounded. Using the fact that $F_{p}=\hat{F}_{p}[4$, section 25.1$]$, it follows that $F_{p}^{n}$ is also self-dual. Thus $\hat{F}_{p}^{n}$ is divisible, since $F_{p}$ is divisible. We use Proposition 2.3 to discuss the case when $\mu$ is infinite. $M_{b}\left(\mu, F_{p}\right)$ contains the compact open subgroup $M\left(\mu, J_{p}\right)$, and the quotient group is $Z_{p}^{\infty}(\lambda)$. Thus $M_{b}\left(\mu, F_{p}\right)$ contains the compact open subgroup $M\left(\lambda, J_{p}\right)=M\left(\mu, J_{p}\right)^{\perp}$, since $Z_{p}^{\infty \wedge}(\lambda)=M\left(\lambda, J_{p}\right)$. The quotient group $M_{b}^{\wedge}\left(\mu, F_{p}\right) \bmod \left(M\left(\lambda, J_{p}\right)\right)$ must be isomorphic to $Z_{p}^{\infty}(\mu)=M^{\wedge}\left(\mu, J_{p}\right)$, since $M\left(\lambda, J_{p}\right)$ is the annihilator in $M_{b}\left(\mu, F_{p}\right)$ of $M\left(\mu, J_{p}\right)$. We will show that this is a contradiction if $M_{b} \widehat{(}\left(\mu, F_{p}\right)$ is assumed to be divisible. $M_{b}\left(\mu, F_{p}\right)$ is torsion-free by [4, Theorem 24.23, p. 384]. If $M_{b}\left(\mu, F_{p}\right)$ is divisible, it follows from [4, Theorem A.17, p. 446] that $M_{b}^{\widehat{ }}\left(\mu, F_{p}\right)$ is the minimal divisible extension of $M\left(\lambda, J_{p}\right)$ $=M\left(\mu, J_{p}\right)^{\perp}$. Thus $M_{b}\left(\mu, F_{p}\right)=M_{b}\left(\lambda, F_{p}\right)$ by definition. We have on the one 
hand that the quotient group $M_{b}\left(\mu, F_{p}\right) \bmod M\left(\lambda, J_{p}\right)$ is $Z_{p}^{\infty}(\mu)$, and on the other hand it is $Z_{p}^{\infty}(v), v \geqq 2^{\lambda}$, by Proposition 2.3. This yields the contradiction

$$
\mu=v \geqq 2^{\lambda}>\lambda \geqq 2^{\mu} .
$$

2.8. Definitions. The minimal divisible extension $Q_{d}$ of the integers $Z$ is the discrete rationals. $Q_{d}(\mu)$ denotes the weak direct sum $L\left(\mu, Q_{d}, e\right)$, and $M\left(\mu, \hat{Q}_{d}\right)$ denotes the dual group $L\left(\mu, \hat{Q}_{d}, \hat{Q}_{d}\right)$. This is abbreviated to $\hat{Q}_{d}$ if $\mu$ has only one element.

2.9. Proposition. $M\left(\mu, \hat{Q}_{d}\right)$ is a compact connected group, and every compact connected group is a quotient group of $M\left(\mu, \hat{Q}_{d}\right)$ for some $\mu$.

Proof. $M\left(\mu, \hat{Q}_{d}\right)$ is connected because $Q_{d}(\mu)$ is torsion-free [4, Theorem 24.25, p. 385]. Proposition 1.8 shows that every compact connected group is a quotient group of a group $A$, where $\hat{A}$ is torsion-free and divisible. Hence $\hat{A}=Q_{d}(\mu)$, so $A=M\left(\mu, \hat{Q}_{d}\right)$.

2.10. Counterexample. A connected and simply connected locally compact abelian group $G$ need not be locally connected, even if it is compact and torsionfree. Take $G=\hat{Q}_{d}$. It follows from [8, p. 169], or [10, p. 93] that $\hat{Q}_{d}$ fails to be locally connected, and $[3$, p. 878$]$ shows that $\hat{Q}_{d}$ is simply connected. $\hat{Q}_{d}$ is also torsion-free, since the existence of a finite subgroup of $\hat{Q}_{d}$ would imply the existence of a finite quotient group of the divisible group $Q_{d}$.

REMARK. The fact that $\hat{Q}_{d}$ fails to be locally connected may be established fairly directly by using Ascoli's theorem to show that every locally connected group satisfies property $\mathbf{P}$ (defined below). $\hat{Q}_{d}$ fails to have property $\mathbf{P}$, as can be seen by examining the neighborhood in $\hat{Q}_{d}$ consisting of all elements which map $1 \in Q_{d}$ into a small neighborhood $T_{\varepsilon}$ of the identity in $T$. We say that $G$ has property $\mathrm{P}$ if, for every neighborhood $N$ of $e \in G$, there is a compact subset $K_{N}$ of $\hat{G}$ such that every character $\alpha \notin K_{N}$ satisfies $\alpha(N)=T$. (Briefly, every 0 -neighborhood is mapped onto $T$ by an $\infty$-neighborhood.)

2.11. Definition. Let $p$ be a prime, and $Z_{p}$ the p-element cyclic group. $Z_{p}(\mu)$ denotes the weak direct sum $L\left(\mu, Z_{p}, e\right)$, and $M\left(\mu, Z_{p}\right)$ denotes the strong direct product $L\left(\mu, Z_{p}, Z_{p}\right)$. Let $Z_{p}(\lambda, \mu)$ denote the direct sum $Z_{p}(\lambda)+M\left(\mu, Z_{p}\right)$. Then $Z_{p}(\lambda, \mu)$ is a Z-group, since $\left(Z_{p}, e\right)$, and $\left(Z_{p}, Z_{p}\right)=\left(\hat{Z}_{p}, e^{\perp}\right)$ are $Z$-pairs.

2.12. Proposition. Let $G$ be a group such that every element has order $p$. Then $G$ is a Z-group.

Proof. If every nonzero element has order $p$, then $p$ must be a prime. Braconnier shows in [2, Theorem 2, p. 41] that $G=Z_{p}(\lambda, \mu)$. (See also [4, section 25.29, p. 417].)

2.13. Proposition. Let $G$ be the additive group of a locally compact field which is not connected. Then $G$ is a Z-group.

Proof. It is shown in $[9, \mathrm{Vol} . \mathrm{I}, \mathrm{p} .189]$ that any such group is $F_{p}^{n}$ or $Z_{p}(\omega, \omega)^{n}$, where $\omega$ is the first infinite cardinal. 
2.14. Counterexample. If $A$ and $B$ are algebraically isomorphic and topologically isomorphic, they need not be isomorphic, even if $A$ is second countable, and $B=A^{2}$. Take $A=F_{p}$. We begin by noting that $Z_{p}(\omega, \omega)$ is isomorphic as a topological group to $Z_{p}(\omega, \omega)+Z_{p}(\omega, \omega)$. This is because

$$
\begin{aligned}
Z_{p}(\omega, \omega)+Z_{p}(\omega, \omega) & =Z_{p}(\omega)+Z_{p}(\omega)+M\left(\omega, Z_{p}\right)+M\left(\omega, Z_{p}\right) \\
& =Z_{p}(\omega+\omega)+M\left(\omega+\omega, Z_{p}\right) \\
& =Z_{p}(\omega)+M\left(\omega, Z_{p}\right)=Z_{p}(\omega, \omega) .
\end{aligned}
$$

Both $F_{p}$ and $Z_{p}(\omega, \omega)$ are unions of totally disconnected compact metric spaces, and a topological homeomorphism can be constructed by using the usual expansion for elements of $p$-adic and $p$-series fields. Thus we have, topologically,

$$
F_{p} \simeq Z_{p}(\omega, \omega) \cong Z_{p}(\omega, \omega)^{2} \simeq F_{p}^{2} .
$$

Algebraically, we see that $F_{p}$ is an uncountable torsion-free divisible group, so $F_{p}=Q_{d}(\mu)$. Thus $F_{p}=F_{p}^{2}$, since they both have the same cardinality. It is not difficult to see that the homeomorphic automorphisms of $F_{p}^{2}$ act transitively on the compact open subgroups of $F_{p}^{2}$. (See Proposition 5.7 below.) Thus a topological group isomorphism between $F_{p}$ and $F_{p}^{2}$ would establish a topological isomorphism between $J_{p}$ and $J_{p}^{2}$. This would in turn entail an isomorphism between $Z_{p}^{\infty}$ and $\left(Z_{p}^{\infty}\right)^{2} . Z_{p}^{\infty}$ is however different from $\left(Z_{p}^{\infty}\right)^{2}$, since the vector space subgroups of elements of order $p$ are different.

\section{Connectivity, divisibility, and torsion.}

3.1. Definitions. Additive notation is used for all the groups of this section. Given a prime number $p$, we say that $G$ is a topological $p$-group if $p^{n} x \rightarrow 0$ for every $x \in G$. $G$ is a topological torsion group if $(n !) x \rightarrow 0$ for every $x \in G$, and $G$ is topologically torsion-free if $(n !) x \rightarrow 0$ implies $x=0$.

We give alternative characterizations of topological torsion groups and topological p-groups in Propositions 3.15 and 3.17.

3.2. Proposition. Let $\alpha$ be a continuous homomorphism, and suppose $G$ is a topological torsion group (p-group). Then $\alpha(G)$ is a topological torsion group ( $p$ group). A discrete group is a topological torsion group (p-group)if and only if it is a torsion group ( $p$-group), and is topologically torsion-free if and only if it is torsion-free.

Proof. The proofs are all immediate.

3.3. Proposition. Suppose $G$ is a compact group. If $G$ is totally disconnected, then $G$ is a topological torsion group.

Proof. Let $U$ be an arbitrary neighborhood of the identity. Then $U$ contains a compact open subgroup $H$ of $G$, since every totally disconnected group contains arbitrarily small compact open subgroups. The quotient group $G / H$ is a compact discrete group, and therefore $(n !) x$ is eventually in $H$ for every $x \in G$. Thus $(n !) x \rightarrow 0$, so $G$ is a topological torsion group. 
It is not difficult to prove the converse of the above proposition. Assuming $G$ compact, we need only observe that every character annihilates the identity component of $G$, since the $\theta \in T$ such that $(n !) \theta \rightarrow 0$ constitute a proper subset of the circle $T$. Examining this a bit more closely, we see that we have an unbounded subset $S=\{n !\} \subset \hat{T}$, and that there is a dense set of $\theta \in T$ such that $\{n ! \theta\}$ is dense in $T$. We can conclude that the set $\{\theta \in T:(n !) \theta \rightarrow 0\}$ is a proper subset of $T$, and we can also conclude that the sequence $\{n !\} \subset \hat{T}$ cannot have a subsequence which is pointwise convergent on $T$. It turns out that this behavior generalizes very nicely, so we make a digression to prove a theorem which guarantees the existence of certain dense subsets of a connected group. The main discussion will resume at Lemma 3.14.

3.4. Definition. Let $N$ be a neighborhood of the identity in a locally compact abelian group $G$. We say that $N$ is quasi-connected in $G$ if, for every cyclic group $Z_{m}$, there is a compact subset $K\left(N, Z_{s \geqq m}\right)$ of $\hat{G}$ such that $\alpha \notin K\left(N, Z_{s \geqq m}\right)$ implies that $\alpha(N)$ contains a neighborhood of $Z_{s}$ for some $s \geqq m$. $G$ is said to be locally quasiconnected if there is a quasi-connected base at the identity. Note that every discrete group is locally quasi-connected, since the dual is compact. A simple characterization of locally quasi-connected groups is stated in the remark which follows Proposition 3.10.

3.5. Lemma. Suppose $A$ is locally quasi-connected, and $B=\pi(A)$ is a quotient group. Then $B$ is locally quasi-connected.

Proof. If $N_{B}$ is a neighborhood of the identity in $B$, then $N_{B}$ is the image of a quasi-connected neighborhood $N_{A}$, since quotient group surjections are open mappings. Let $\pi^{t}: \hat{B} \rightarrow \hat{A}$ denote the transpose of $\pi$. Then $\pi^{t}$ is a homeomorphic isomorphism from $\hat{B}$ onto the annihilator in $\hat{A}$ of $\pi^{-1}(0)$, and $\pi^{t}(\beta)$ fails to map $N_{A}$ onto a neighborhood of $Z_{s}$ if $\beta$ fails to map $N_{B}$ onto a neighborhood of $Z_{s}$. Thus the characters which fail to map $N_{B}$ onto a neighborhood of some $Z_{s}, s \geqq m$, are contained in the compact set $\left(\pi^{t}\right)^{-1}\left(K\left(N_{A}, Z_{s \geqq m}\right) \cap\left[\pi^{-1}(0)\right]^{\perp}\right)$.

3.6. Lemma. Suppose $G=A+B$ is a direct sum of two groups. Then $G$ is locally quasi-connected if and only if each summand is locally quasi-connected.

Proof. Suppose that the summands $A$ and $B$ are both locally quasi-connected. If $N_{A}$ and $N_{B}$ are quasi-connected sets in the respective summands, then we show that $N_{A} \times N_{B}$ is quasi-connected, provided $N_{A}$ and $N_{B}$ each contain the identity of the summand. A character $\alpha \times \beta$ maps $N_{A} \times N_{B}$ onto the product $\alpha\left(N_{A}\right) \cdot \beta\left(N_{B}\right)$, the product being taken in the circle $T$. Thus $\alpha \times \beta$ maps $N_{A} \times N_{B}$ onto a neighborhood of $Z_{s}$ if either $\alpha\left(N_{A}\right)$ or $\beta\left(N_{B}\right)$ is a neighborhood of $Z_{s}$, and the set of characters $\alpha \times \beta$ which fail to map $N_{A} \times N_{B}$ onto a neighborhood of some $Z_{s}, s \geqq m$, is contained in the compact set $K\left(N_{A}, Z_{s \geqq m}\right) \times K\left(N_{B}, Z_{s \geqq m}\right)$. The converse follows from the previous Lemma 3.4.

Counterexample 2.10 shows that the group $\hat{Q}_{d}$ is connected but not locally connected. We now show that $\hat{Q}_{d}$ is locally quasi-connected. 
3.7. Lemma. Let $r$ and $s$ be positive integers, and let $N_{r}$ be the set of all characters on $Q_{d}$ which map $1 / r$ into a fixed $\varepsilon$-neighborhood $T_{\varepsilon}$ of the identity in $T$. Then $1 / r s\left(N_{r}\right)$ contains a neighborhood of $Z_{s}$.

Proof. Any character which is defined on the set of integer multiples of $1 / r s \in Q_{d}$ can be extended to an element of $\hat{Q}_{d}$. For any $z \in T$, there exists a character $\alpha$, defined on the subgroup generated by $1 / r s$, such that $\alpha(1 / r s)=z$. Thus there are elements of $N_{r}$ which map $1 / r s$ onto any sth root of $T_{\varepsilon}$.

3.8. Proposition. $\hat{Q}_{d}$ is locally quasi-connected.

Proof. We consider the set $S$ of neighborhoods of the identity in $\hat{Q}_{d}$ which have the form $N_{r}$; that is, $N_{r} \in S$ if there exists an $\varepsilon$ such that $N_{r}$ consists of all characters which map $1 / r$ into $T_{\varepsilon}$. Due to the algebraic conditions on characters, $S$ forms a basis at $e$ in $\hat{Q}_{d}$. We will show that every element of $S$ is a quasi-connected set. Given a positive integer $m$ and a neighborhood $N_{r} \in S$, we wish to know which characters $p / q \in Q_{d}$ fail to map $N_{r}$ onto a neighborhood of $Z_{k}$ for some $k \geqq m$. It suffices to show that the set of such $p / q$ which are positive is a finite set. We assume $p$ and $q$ positive and relatively prime. Let $j$ be the greatest common divisor of $q$ and $r$ so that $r=j t, q=j s$, with $s$ and $t$ relatively prime. Note that $1 / q=t / r s$. The integers $r$ and $m$ will be fixed throughout the rest of the discussion. Turning our attention to $q=j s$, we consider two cases.

Case I. $s<m$. There are only finitely many divisors $j$ of $r$, and there are only finitely many $s<m$, so our discussion of Case I will involve only finitely many $q$.

Case II. $s \geqq m$.

For Case I, we show that $1 / q\left(N_{r}\right)$ contains a neighborhood of the identity in $T$. It suffices to show that $1 / q\left(N_{r}\right)$ contains the $t$ th power of some neighborhood of the identity in $T$. By Lemma 3.7, $1 / r s\left(N_{r}\right)$ contains a neighborhood of the identity in $T$, and we have

$$
1 / q\left(N_{r}\right)=t / r s\left(N_{r}\right)=\left[1 / r s\left(N_{r}\right)\right]^{t} .
$$

Since $1 / q\left(N_{r}\right)$ contains a neighborhood of the identity, it follows that $p / q\left(N_{r}\right)=T$ whenever $p$ is large enough.

For Case II, we know by Lemma 3.7 that $1 / r s\left(N_{r}\right)$ contains a neighborhood of $Z_{s}$. The same is true for $1 / q\left(N_{r}\right)=t / r s\left(N_{r}\right)$, since $t$ is relatively prime to $s$. Moreover, $p$ is relatively prime to $s$, since $p$ is relatively prime to $q=j s$. Thus $p / q\left(N_{r}\right)$ contains a neighborhood of $Z_{s}$. Combining Case I and Case II, we see that there are only finitely many $p / q$ which fail to map $N_{r}$ onto a neighborhood of $Z_{s}$ for some $s \geqq m$.

3.9. Corollary. $M\left(\mu, \hat{Q}_{d}\right)$ is locally quasi-connected.

Proof. There are basic neighborhoods of the form $N_{F} \times M\left(\mu-F, \hat{Q}_{d}\right)$, where $F$ is a finite subset of $\mu$ and $N_{F}$ is a basic neighborhood in $M\left(F, \hat{Q}_{d}\right)$. If $\alpha \times \beta$ is a character which fails to map $N_{F} \times M\left(\mu-F, \hat{Q}_{d}\right)$ onto a neighborhood of $Z_{s}$, then $\beta$ must fail to map $M\left(\mu-F, \hat{Q}_{d}\right)$ onto a neighborhood of $Z_{s} . M\left(\mu-F, \hat{Q}_{d}\right)$ is a 
compact connected group, so it follows that $\beta=e$. Thus the characters $\alpha \times \beta$ under consideration have $\beta$ contained in a finite set; $\alpha$ is also contained in a finite set, since $N_{F}$ is a basic set in $M\left(F, \hat{Q}_{d}\right)$, and the above Proposition 3.8 together with Lemma 3.6, shows that $M\left(F, \hat{Q}_{d}\right)$ is locally quasi-connected.

\subsection{Proposition. Every connected group is locally quasi-connected.}

Proof. Every connected group is a direct sum $R^{n}+K$, where $K$ is a compact connected group [4, Theorem 9.14, p. 95]. Using Lemma 3.6, it is not difficult to see that Euclidean $n$-space is locally quasi-connected. Thus another application of Lemma 3.6 shows that we may restrict our attention to a compact connected group $K$. Proposition 2.9 shows that any such $K$ is a quotient group of $M\left(\mu, \hat{Q}_{d}\right)$ and Corollary 3.9 shows that $M\left(\mu, \hat{Q}_{d}\right)$ is locally quasi-connected. Thus $K$ is locally quasi-connected, by Lemma 3.5.

REMARK. Using the fact that an open subgroup of a locally quasi-connected group is a locally quasi-connected group, it can be shown that $G$ is locally quasiconnected if and only if the identity component $G_{0}$ is open. An open identity component is always a topological direct summand [4, p. 395]. Thus $G$ is locally quasi-connected if and only if $G=G_{0}+D$, where $D$ is a discrete group.

3.11. TheOREM. Let $G$ be locally quasi-connected, and let $S$ be a subset of $\hat{G}$ which intersects every neighborhood of $\infty$. Then $G_{S}$ is dense in $G$, where

$$
G_{S}=\{g \in G: g(S) \text { is dense in } T\} .
$$

Proof. Let $N$ be a neighborhood of the identity in $G$, and let $\alpha$ be a character. Then $\alpha(N)$ is $\varepsilon$-dense in the circle if and only if $\alpha(N+x)$ is $\varepsilon$-dense in the circle for every $x \in G$. Thus, given $V \neq \varnothing$ open in $G$ and $M \neq \varnothing$ open in the circle, the hypotheses allow us to choose an $\alpha \in S$ such that $\alpha(V) \cap M \neq \varnothing$, since every $V$ contains a translate of some quasi-connected neighborhood of the identity. Note that $\alpha(V) \cap M \neq \varnothing$ is the same as $\alpha^{-1}(M) \cap V \neq \varnothing$. Here $\alpha^{-1}(M)$ denotes the inverse image under $\alpha$ of the set $M$, and is not intended to convey any suggestion of the group-theoretic inverse of $\alpha$.

Given an open subset $U$ of $G$, we wish to prove that $U$ contains some element of $G_{S}$. We first choose an open set $U_{1}$ such that $\bar{U}_{1}$ is compact and contained in $U$.

We will now work our way around the circle. The subgroup $Z_{n}$ of $T$ determines $n$ congruent open intervals in the circle; we let $T_{n, j}$ denote the $j$ th such interval. Beginning with $Z_{3}$, we first construct an open set $U_{3}$ such that $\bar{U}_{3} \subset U_{1}$, and $x(S)$ is $1 / 3$-dense in $T$ for every $x \in U_{3}$. We denote by $\alpha_{31}$ some element of $S$ such that $\alpha_{31}^{-1}\left(T_{31}\right) \cap U_{1} \neq \varnothing$, and we let $U_{31}=\alpha_{31}^{-1}\left(T_{31}\right) \cap U_{1}$. In other words, we have chosen $\alpha_{31} \in S$ such that $U_{31}$ is not empty, where $U_{31}=\alpha_{31}^{-1}\left(T_{31}\right) \cap U_{1}$. Choose $\alpha_{32} \in S$ such that $U_{32}=\alpha_{32}^{-1}\left(T_{32}\right) \cap U_{31}$ is not empty, and choose $\alpha_{33} \in S$ such that $U_{33}=\alpha_{33}^{-1}\left(T_{33}\right) \cap U_{32}$ is not empty. Let $U_{3}$ be an open set such that $\bar{U}_{3} \subset \bigcap_{1}^{3} U_{3 j}$. Then for $x \in U_{3}$, we have $x\left(\alpha_{3 j}\right) \in T_{3 j}$, so $x(S)$ is $1 / 3$-dense in $T$. 
The general procedure is clear from the above. Given $U_{n}$, we choose $\alpha_{n+1,1} \in S$ such that

$$
U_{n+1,1}=\alpha_{n+1,1}^{-1}\left(T_{n+1,1}\right) \cap U_{n}
$$

is not empty. Then for $j \leqq n$, we choose $\alpha_{n+1, j+1} \in S$ such that

$$
U_{n+1, j+1}=\alpha_{n+1, j+1}^{-1}\left(T_{n+1, j+1}\right) \cap U_{n+1, j}
$$

is not empty. Again utilizing the regularity of locally compact spaces, we choose $U_{n+1}$ an open set such that $\left[U_{n+1}\right]^{-} \subset \bigcap_{1}^{n+1} U_{n+1, j}$. This implies that $\left[U_{n+1}\right]^{-} \subset U_{n}$, since $U_{n+1,1} \subset U_{n}$. Recalling that $\bar{U}_{1}$ is compact, we let $x$ be an element of the nonempty intersection $\bigcap U_{n}$. Then $x \in U$, and $x \in G_{S}$, since $x\left(\alpha_{n j}\right) \in T_{n j}$.

3.12. Corollary. Let $G$ be a connected group, and let $\left\{\alpha_{n}\right\}$ be a sequence in $\hat{G}$ such that $\infty$ is a cluster point. Let $G^{0}\left\{\alpha_{n}\right\}$ denote the set of all $x \in G$ such that $x\left(\alpha_{n}\right) \rightarrow 0$, where 0 is the identity in $T$. Then $G^{0}\left\{\alpha_{n}\right\}$ is a subgroup of $G$, and $G^{0}\left\{\alpha_{n}\right\}$ has a dense complement in $G$.

Proof. It is clear that $G^{0}\left\{\alpha_{n}\right\}$ is a subgroup of $G$, and the theorem shows that the complement contains a dense set, since $x\left(\alpha_{n}\right)$ cannot converge to 0 if $x\left(\alpha_{n}\right)$ is dense in $T$.

3.13. Corollary. Let $G$ be a connected group, and let $\left\{\alpha_{n}\right\}$ be a sequence in $\hat{G}$ which converges to $\infty$. Then there exists a subnet of $\left\{\alpha_{n}\right\}$ which is pointwise convergent on $G$, but no subsequence of $\left\{\alpha_{n}\right\}$ is pointwise convergent.

Proof. The pointwise topology on $\hat{G}$ is the product topology of the circle taken as many times as there are points in $G$. Thus we are dealing with a compact space, and convergent subnets exist. Given any subsequence of $\left\{\alpha_{n}\right\}$, there is a dense set of points in $G$ at which convergence fails.

REMARK. Since the proof of Theorem 3.11 uses arguments which depend only on $\varepsilon$-dense sets (rather than neighborhoods of arbitrarily large cyclic groups), the result is also valid for groups such as $F_{p}^{n}$.

Returning from our digression on locally quasi-connected groups, we characterize topological torsion groups.

3.14. Lemma. Suppose $K$ is compact. Then $K$ is a topological torsion group if and only if $\hat{K}$ is totally disconnected, and $K$ is a topological p-group if and only if $\hat{K}$ is a discrete p-group.

Proof. If $K$ is totally disconnected, then Proposition 3.3 shows that $K$ is a topological torsion group. Suppose $K$ is a topological torsion group. Then every character maps $K$ into a subgroup $T^{0}\{n$ ! $\}$ of $T$ consisting of those $\theta \in T$ such that $(n !) \theta \rightarrow 0$. Corollary 3.12 shows that this subgroup of $T$ is not all of $T$. Thus every character annihilates the identity component of $K$, so $K$ is totally disconnected. Suppose $K$ is a topological $p$-group. Corollary 3.12 shows that $T^{0}\left\{p^{n}\right\}$ is a 
proper subgroup of $T$, and so once again the identity component is annihilated by every character, since the image of the identity component is a connected subgroup of $T$. Thus $K$ is totally disconnected, and so $K$ contains arbitrarily small compact open subgroups. The corresponding quotient groups are compact discrete $p$ groups, by Proposition 3.2. Thus $\hat{K}$ has arbitrarily large subgroups which are discrete $p$-groups, i.e., every element of $\hat{K}$ is contained in a $p$-group.

3.15. THEOREM. The following conditions are equivalent:

(1) $(n !) x \rightarrow 0$ for every $x \in G$.

(2) Every character on $G$ has proper range, and every character on $\hat{G}$ has proper range.

(3) $G$ and $\hat{G}$ are both totally disconnected.

(4) Every character on $G$ has range contained in the rational circle.

(5) $G$ has arbitrarily small compact open subgroups such that the quotient groups are discrete torsion groups.

Proof. The proofs $(1) \Rightarrow(2) \Rightarrow(3)$ are the same as the corresponding proofs for the compact case, all that is necessary is to observe that $T^{0}\{n !\}$ is a proper subgroup of $T$ (see Lemma 3.14). Assuming (3), we note that every element of $G$ is contained in some compact open subgroup $K$ such that $K$ is totally disconnected [4, Corollary 24.18, p. 383]. Lemma 3.14 shows that each totally disconnected $K$ is a topological torsion group. Thus every element of $\hat{G}$ maps $K$ onto a compact proper subgroup of $T$; the only such subgroups of $T$ are the finite subgroups. Thus the range of any character is contained in the union of the finite subgroups of $T$. Assuming condition (4), we see that conditions (2) and (3) may also be assumed, since $(4) \Rightarrow(2)$. Thus $\hat{G}$ has arbitrarily large compact open subgroups $K^{\perp}$ which are totally disconnected, by (3). Let $x$ be an element of $G$ and let $K$ be a compact open subgroup of $G$. Then we may again apply the argument which shows that $x\left(K^{\perp}\right)$ is a finite subgroup of the circle. It follows that there is an $n$ such that $n x \in K=K^{\perp \perp}$. Thus $G / K$ is a discrete torsion group, and consequently (4) $\Rightarrow$ (5). The arguments used in Proposition 3.3 can be used to show (5) $\Rightarrow$ (1).

3.16. COROLlaRY. $G$ is a topological torsion group iff $\hat{G}$ is a topological torsion group.

Proof. Condition (3) of the theorem (and also conditions (2) and (4)) holds for $G$ if and only if it holds for $\hat{G}$.

3.17. Proposition. The following conditions are equivalent:

(1) $G$ is a topological p-group.

(2) Every character on $G$ has range contained in $Z_{p}^{\infty}$.

(3) $G$ has arbitrarily small compact open subgroups such that the quotient groups are discrete p-groups.

(4) Every element of $G$ is contained in a group which is a continuous image of $M\left(\mu, J_{p}\right)$. 
Proof. The equivalence of (1), (2), and (3) follows along the lines of Theorem 3.15 and Lemma 3.14. We need only note that $T^{0}\left\{p^{n}\right\}$ is a proper subgroup of $T$, and it follows that we have a topological torsion group. If $p^{n} x \rightarrow 0$, then every character on $\hat{G}$ maps compact open subgroups of $\hat{G}$ onto finite subgroups of $T$ which are discrete $p$-groups. The union of such subgroups of $T$ is precisely $Z_{p}^{\infty}$. We now assume that conditions (1)-(3) are equivalent. Given condition (1), it follows from Lemma 3.15 that every element of $G$ is contained in a compact open subgroup $K$ such that $K$ is a topological $p$-group. Lemma 3.14 shows that $\hat{K}$ is a subgroup of $Z_{p}^{\infty}(\mu)=E(\hat{K})$, and so it follows that $K$ is a quotient group of the dual group $M\left(\mu, J_{p}\right)$. Thus (1) $\Rightarrow(4) . M\left(\mu, J_{p}\right)$ is a topological $p$-group, and so Proposition 3.2 shows that $(4) \Rightarrow$ (1).

3.18. Corollary. $G$ is a topological p-group if and only if $\hat{G}$ is a topological p-group.

Proof. Condition (2) of the theorem holds for $G$ if and only if it holds for $\hat{G}$.

Using the above characterizations of topological torsion groups and topological p-groups, the decomposition theorem of [2, p. 49] can be reformulated as an assertion that every topological torsion group is a local direct product of topological p-groups. We state this as Theorem 3.21.

3.19. Definition. Let $p$ be a prime. The subgroup $A_{p}$ of $A$ consisting of those $x$ such that $p^{n} x \rightarrow 0$ is called the p-summand of $A$.

3.20. LemmA. Let $K$ be a compact topological torsion group. Then the p-summands $K_{p}$ are closed, and $K$ is the strong direct product of its p-summands $K_{p}$.

Proof. Lemma 3.14 shows that $K$ is compact, and [4, Theorem 24.26, p. 385] shows that $\hat{K}$ is a discrete torsion group. Thus $\hat{K}$ is a weak direct sum of discrete p-groups [5, p. 5]. It follows from Lemma 3.14, and [2, Theorem 1, p. 10 and Proposition 2, p. 48] that $K$ is the strong direct product of the duals of these discrete $p$-groups, and that each $K_{p}$ is isomorphic to one of these $p$-group duals, since $K_{p}$ consists of the subgroup which annihilates all elements with order relatively prime to $p$. In particular, each $K_{p}$ is closed.

3.21. TheOREM. Let $A$ be a topological torsion group. Then the p-summands $A_{p}$ are closed, and $A$ is a local direct product of the topological p-groups $A_{p}$.

Proof. The $p$-summands are closed, since every element of $A$ is contained in a compact open subgroup (Theorem 3.15), and the result is valid for compact groups (Lemma 3.20). The theorem of [2, p. 49] establishes the result in general; we note that condition (3) of Theorem 3.15 shows that $G$ is a topological torsion group if and only if $G$ and $\hat{G}$ are both totally disconnected, whereas condition (4) of Proposition 3.17 shows that we may use the Definition 3.1 for topological p-group, rather than Braconnier's definition [2, Definition 1, p. 46].

This finishes our results on topological torsion groups. We proceed with a discussion of topologically torsion-free groups. (See Definition 3.1.) 
3.22. Proposition. $\hat{Q}_{d}$ fails to be topologically torsion-free.

Proof. Let $K$ be the annihilator of the integers. Then $\hat{K}$ is the rational circle, so $K$ is a topological torsion group by Corollary 3.16. Thus there are nonzero $x \in \hat{Q}_{d}$ such that $(n !) x \rightarrow 0$.

3.23. Corollary. $M\left(\mu, \hat{Q}_{d}\right)$ fails to be topologically torsion-free.

Proof. There exist continuous injections from $\hat{Q}_{d}$ into $M\left(\mu, \hat{Q}_{d}\right)$. Thus $M\left(\mu, \hat{Q}_{d}\right)$ contains nonzero elements $x$ such that $(n !) x \rightarrow 0$.

3.24. Proposition. The only compact group which is topologically torsion-free is the one-element group.

Proof. If $K$ is topologically torsion-free, then $K$ is torsion-free. If $K$ is also nontrivial, then $K$ is a local direct product $L\left(\mu, G_{x}, G_{x}\right)$, where either $G_{x}=\hat{Q}_{d}$, or $G_{x}$ is a topological torsion group $J_{p}$. (See Definitions 2.1, 2.8, and also [4, Theorem 25.8, p. 406].) We have shown that all the possible constituents of $K$ are groups which fail to be topologically torsion-free (Corollary 3.23). Thus $K$ has only one element.

3.25. THEOREM. $G$ is connected if and only if $\hat{G}$ is topologically torsion-free.

Proof. If $G$ is connected, then $G=R^{n}+K$, where $K$ is compact and connected. Thus $G=\hat{R}_{n}+\hat{K}$, where $\hat{K}$ is discrete and torsion-free, so $\hat{G}$ is topologically torsionfree [4, Theorem 24.25 , p. 385 ]. Conversely, assume $\hat{G}$ is topologically torsion-free. Then $\hat{G}=R^{n}+H$, where $H$ has a compact open subgroup $K[4$, Theorem 24.30, p. 389]. $H$ must be discrete, since Proposition 3.24 shows that $K$ has only one element. Thus $G=R^{n}+J$, and $J$ is connected because $\hat{J}=\hat{G} / J^{\perp}$ is the torsion-free discrete group $H$.

In $\S 5$ we will show that $G$ is torsion-free if and only if $\hat{G}$ contains a divisible dense subgroup. For now we establish results on torsion-free groups which will be quite useful in the discussion of endomorphism-simple groups.

3.26. Proposition. Suppose $G$ is torsion-free and has compact identity component. Then $G$ is an open subgroup of a Z-group.

Proof. The minimal divisible extension $E(G)$ is torsion-free [4, Theorem A.16, p. 446]. Thus $E(G)=A+B+C$ where $A=M\left(\mu, \hat{Q}_{d}\right), B=Q_{d}(\lambda)$, and $C$ is a local direct product $L\left(X, G_{p}, K\left(G_{p}\right)\right)$, where $X$ is a set of prime numbers, and $\left(G_{p}, K\left(G_{p}\right)\right)$ is for each $p \in X$, the $Z$-pair $\left(M_{b}\left(\nu, F_{p}\right), M\left(\nu, J_{p}\right)\right)$. Here $\nu$ depends upon $p$. (See [7, p. 940], and also [4, section 25.33, p. 421].) $E(G)$ is thus a $Z$-group, and $G$ is by definition open in $E(G)$.

3.27. Proposition. Let $G$ be a group such that $\hat{G}$ is torsion-free. Then $G=R^{n}$ $+Q_{d}(\mu)+B$, where $B$ is the closed subgroup consisting of all compact elements. (See [4, Theorem 9.10, p. 92].) The identity component $B_{0}$ of $B$ is compact, and the quotient group $A=B / B_{0}$ is a topological torsion group. 
Proof. Since $\hat{G}$ is torsion-free, the identity component is a direct summand [4, Section $25.30 \mathrm{c}$, p. 418]. Thus $\hat{G}=R^{n}+K+C$, where $K$ is compact connected, and $C$ is totally disconnected. $K^{\perp}=Q_{d}(\mu)$ by [4, Theorem 25.8, p. 406]. Dualizing the equation $\hat{G}=R^{n}+K+C$, we arrive at $G=R^{n}+Q_{d}(\mu)+B$, where $B$ is the annihilator of the identity component of $\hat{G}[4$, Theorem 24.17 , p. 382]. $B$ cannot contain any isomorphic copies of $R^{n}$, because $R^{n}$ has no nonzero compact elements. Hence $B_{0}$ must be compact. The dual of $A$ must be a subgroup of the totally disconnected group $C$. Thus $A$ and $\hat{A}$ are both totally disconnected, so $A$ is a topological torsion group by Theorem 3.15 .

\section{Homogeneous groups.}

4.1. Definitions. $G$ is endomorphism-simple if no proper closed subgroup is mapped into itself under every continuous endomorphism. $G$ is homogeneous if the group of homeomorphic automorphisms acts transitively on the nonzero elements of $G$.

\subsection{Proposition. Endomorphism-simple groups occur in dual pairs.}

Proof. $H$ is invariant under the endomorphism $h$ if and only if $H^{\perp}$ is invariant under the transpose $h^{t}$. Thus $A$ is endomorphism-simple if and only if $\hat{A}$ is endomorphism-simple.

4.3. CountereXample. The dual of a homogeneous group need not be homogeneous. Take $G=Q_{d}$. Then $G$ is homogeneous, since every nonzero element is part of a vector space basis, and any linear map which carries a basis onto a basis is a homeomorphic automorphism. $\hat{Q}_{d}$, however, is not homogeneous, since a homogeneous group is either a topological torsion group or else is topologically torsion-free. Corollary 3.16 and Proposition 3.24 show that $\hat{Q}_{d}$ satisfies neither of these conditions. Propositions 4.7 and 4.12 together imply that $G=M_{b}\left(\mu, F_{p}\right)$ provides another counterexample.

4.4. Proposition. Suppose $G$ is endomorphism-simple. If $G$ is a torsion-free topological torsion group, then $G$ is isomorphic to an open subgroup of $M_{b}\left(\mu, F_{p}\right)$.

Proof. Theorem 3.21 shows that the $p$-summands of $G$ are closed, and hence $G$ must coincide with some $p$-summand, since each $p$-summand is invariant under continuous endomorphisms. Referring to the proof of Proposition 3.26, we see that $G$ cannot be a $p$-group unless $G$ is an open subgroup of $M_{b}\left(\mu, F_{p}\right)$.

REMARK. The above Proposition 4.4 may be established fairly directly without referring to Mackey's result [7, p. 940]. We simply note that $p \hat{G}$ is a dense subgroup of $\hat{G}$ by Proposition 4.2. If $K$ is a compact open subgroup of $G$, then $K^{\perp}$ is a compact open subgroup of $\hat{G}$, and multiplication by $p$ maps $\hat{G} / K^{\perp}$ onto itself, since $p \hat{G}$ is dense in $\hat{G}$, and $\hat{G} / K^{\perp}$ is discrete. Thus $K=M\left(\mu, J_{p}\right)$, since $\hat{G} / K^{\perp}$ is a divisible discrete $p$-group $Z_{p}^{\infty}(\mu)$, (Corollary 3.16 and Proposition 3.2), and $\hat{K}=\hat{G} / K^{\perp}$. Thus $G$ contains a compact open subgroup $K=M\left(\mu, J_{p}\right)$, and it follows 
from Definition 2.1 and Proposition 2.5 that $E(G)=M_{b}\left(\mu, F_{p}\right)$. Uniqueness of $\mu$ follows from [5, remark (b), p. 11].

4.5. THEOREM. If $A$ is an endomorphism-simple group, then either $A$ belongs to one of the four classes of groups $Z_{p}(\lambda, \mu), R^{n}, Q_{d}(\mu), M\left(\mu, \hat{Q}_{d}\right)$, or else $A$ is isomorphic to some open subgroup of $M_{b}\left(\mu, F_{p}\right)$.

Proof. Case I. $A$ fails to be torsion-free. If some element of $A$ has finite order, then some element of $A$ has prime order $p$. The proof of Proposition 2.12 shows that $A=Z_{p}(\lambda, \mu)$.

Case II. $A$ is torsion-free. In this case the above shows that $\hat{A}$ is torsion-free, otherwise $A=Z_{p}(\mu, \lambda)$, by Proposition 4.2. Since $\hat{A}$ is torsion-free, we know by Proposition 3.27 that $A=R^{n}+Q_{d}(\mu)+B$, where $B$ is the subgroup consisting of all compact elements. $B$ is invariant under all continuous endomorphisms, and is closed. Hence $A=B$, or $B=0$. Assume the latter. Then $A=R^{n}+Q_{d}(\mu)$, and $A_{0}$, the identity component, equals $R^{n}$. $A_{0}$ is closed and invariant under all continuous endomorphisms, hence $A=R^{n}$, or $A=Q_{d}(\mu)$. We are left with the case $A=B$, where the identity component $B_{0}$ is compact (Proposition 3.27). If $B_{0}$ is all of $A$, then $A$ is a compact connected torsion-free group, and so $A=M\left(\mu, \hat{Q}_{d}\right)$ by the proof of Proposition 2.9. The only remaining case is $A=B=B / B_{0}$, that is, $A$ is a topological torsion group. Here Proposition 4.4 shows us that $A$ is an open subgroup of $M_{b}\left(\mu, F_{p}\right)$.

4.6. COROLlaRY. Every endomorphism-simple group is either an open subgroup of a Z-group, or else is $R^{n}$.

Proof. The proof is immediate.

4.7. Proposition. Suppose $G$ is homogeneous. If $G$ is a torsion-free topological torsion group, then $G=M_{b}\left(\mu, F_{p}\right)$. In particular, the dual of $M_{b}\left(\mu, F_{p}\right)$ fails to be homogeneous when $\mu$ is infinite.

Proof. The existence of an automorphism which sends $n y$ onto $x$ shows that $G$ is divisible. Every homogeneous group is endomorphism-simple, and so Proposition 4.4 applies to show that $G$ is an open subgroup of $M_{b}\left(\mu, F_{p}\right)$. It follows from Proposition 2.5 that $G$ is all of $M_{p}\left(\mu, F_{p}\right)$, and from Proposition 2.7 that the dual of $M_{b}\left(\mu, F_{p}\right)$ is not equal to any $M_{b}\left(\lambda, F_{p}\right)$.

4.8. Counterexample. If $G$ is an inverse limit of divisible groups, then $G$ need not be divisible, even if $G$ is torsion-free. Let $G$ be the dual of $M_{b}\left(\mu, F_{p}\right)$ with $\mu$ infinite. The remark following Proposition 4.4 can be used to show that $G$ has arbitrarily small compact open subgroups with divisible quotient groups, whereas Proposition 4.7 shows that $G$ fails to be divisible. It is not difficult to see that $\hat{G}$ divisible implies $G$ torsion-free (see Theorem 5.2 below).

4.9. Proposition. $Z_{p}(\lambda, \mu)$ is homogeneous. 
Proof. We first note that $Z_{p}(\lambda, \mu)$ is isomorphic to $Z_{p}(\lambda-F, \mu+F)$ whenever $F$ is a finite subset of $\lambda$. Given nonzero $f$ and $g$ in $Z_{p}(\lambda, \mu)$, there is a finite subset $F$ of $\lambda$ such that the $\lambda$-components of both $f$ and $g$ are supported on $F$. Consequently we may assume that the $\lambda, \mu$ decomposition of $Z_{p}(\lambda, \mu)$ occurs in such a fashion that $f$ and $g$ both have $\lambda$-components zero. This reduces the problem to the consideration of the homogeneity of $Z_{p}(\phi, \mu)=M\left(\mu, Z_{p}\right)$, since any automorphism $\alpha$ of $M\left(\mu, Z_{p}\right)$ can be extended to $Z_{p}(\lambda)+M\left(\mu, Z_{p}\right)=Z_{p}(\lambda, \mu) .(I \oplus \alpha$, where $I$ is the identity on $Z_{p}(\lambda)$, is such an extension.) Given nonzero $f$ and $g$ in $M\left(\mu, Z_{p}\right)$, the annihilators $f^{\perp}$ and $g^{\perp}$ are hyperplanes in the discrete vector space $Z_{p}(\mu)$. It follows that $f^{\perp \perp}$ may be sent onto $g^{\perp \perp}$ by a homeomorphic automorphism, since the homeomorphic automorphisms of $Z_{p}(\mu)$ act transitively on the set of hyperplanes of $Z_{p}(\mu)$. Both $f^{\perp \perp}$ and $g^{\perp \perp}$ are $p$-element subgroups, and multiplications by nonzero elements of the $p$-element field $Z_{p}$ are homeomorphic automorphisms of $M\left(\mu, Z_{p}\right)$ such that $p$-element subgroups are left invariant. Multiplications by nonzero elements of $Z_{p}$ act transitively on the nonzero elements of a $p$-element group, and it follows that $f$ may be sent onto $g$. Thus $M\left(\mu, Z_{p}\right)$ is homogeneous, and we have shown that this implies the homogeneity of $Z_{p}(\lambda, \mu)$.

4.10. Definitions AND NOtATiON. Regarding $Z_{p}^{\infty}$ as a subgroup of the rationals $\bmod 1$, we use $p^{-n} k$ to denote that element of $Z_{p}^{\infty}$ which is the image of $p^{-n} k \in Q_{d}$. Thus $p^{-n} k=0 \in Z_{p}^{\infty}$ if $p^{-n} k$ is an integer. Characters will frequently be regarded as having their range in $Z_{p}^{\infty} \subset T$ when $J_{p}$ and $Z_{p}^{\infty}$ are regarded as character groups of each other. (See Proposition 3.17, condition (2).) For example, the p-adic integer $1 \in J_{p}$ acts on $p^{-1} \in Z_{p}^{\infty}$ by $1\left(p^{-1}\right)=p^{-1} \in Z_{p}^{\infty}$. We use "characteristic function" notation for certain elements of $M\left(\mu, J_{p}\right)$. If $x \in \mu, C_{x}$ denotes that element of $M\left(\mu, J_{p}\right)$ defined by $C_{x}(y)=0 \in J_{p}$ if $y \neq x, C_{x}(x)=1 \in J_{p}$. We avoid subscripts on subscripts by letting $C_{0}$ denote the characteristic function of $x_{0} \in \mu$. Primed symbols will be used to denote the analogous elements of $Z_{p}^{\infty}(\mu)$. Thus $p^{-n} C_{x}^{\prime}$ denotes that element of $Z_{p}^{\infty}(\mu)$ which maps $x \in \mu$ onto $p^{-n} \in Z_{p}^{\infty}$, and vanishes at all other elements of $\mu . k p^{-n} C_{x}^{\prime}$ denotes the $k$ th additive power of $p^{-n} C_{x}^{\prime}$, and hence is zero if $k$ is an integer divisible by $p^{n} . M\left(\mu, J_{p}\right)$ is always regarded as a subgroup of $M_{b}\left(\mu, F_{p}\right)$. We define the norm of an element $f \in M_{b}\left(\mu, F_{p}\right)$ to be $p^{n}$, where $n$ is the smallest integer such that $p^{n} f \in M\left(\mu, J_{p}\right)$. (See Proposition 2.3.) Note that the norm of an element of $M\left(\mu, J_{p}\right)$ is $\leqq 1$.

4.11. Lemma. Suppose that $f$ is an element of $M\left(\mu, J_{p}\right)$ such that $f(x)=p^{n(x)}$ for every $x \in \mu$, and that norm $f(x)$ attains a maximum at $x_{0} \in \mu$. Then there is a homeomorphic automorphism of $M\left(\mu, J_{p}\right)$ which sends $f$ onto $f\left(x_{0}\right) C_{0}$.

Proof. The automorphism $\alpha$ of $Z_{p}^{\infty}(\mu)$ is defined on a generating subset of $Z_{p}^{\infty}(\mu)$ as follows:

$$
\alpha\left(p^{-n} C_{0}^{\prime}\right)=p^{-n} C_{0}^{\prime}
$$

and, for $x \neq x_{0}$,

$$
\alpha\left(p^{-n} C_{x}^{\prime}\right)=p^{-n} C_{x}^{\prime}+\left[f(x) / f\left(x_{0}\right)\right] p^{-n} C_{0}^{\prime}
$$


Note that $\left[f(x) / f\left(x_{0}\right)\right] \geqq 1$, since the $p$-adic integer $p^{n(x)}$ has norm $\leqq 1$ and exponent $n(x) \geqq 0$. $\alpha$ is a homomorphism of $Z_{p}^{\infty}(\mu)$, and is moreover invertible, since

$$
\alpha^{-1}\left(p^{-n} C_{0}^{\prime}\right)=p^{-n} C_{0}, \quad \alpha^{-1}\left(p^{-n} C_{x}^{\prime}\right)=p^{-n} C_{x}-\left[f(x) / f\left(x_{0}\right)\right] p^{-n} C_{0}^{\prime} .
$$

The action of the transpose $\alpha^{t}$ on $C_{0} \in M\left(\mu, J_{p}\right)$ is given by $\alpha^{t} C_{0}=g$, where $g$ acts on $Z_{p}^{\infty}(\mu)$ by

$$
g\left(p^{-1} C_{0}^{\prime}\right)=p^{-1}, \quad g\left(p^{-1} C_{x}^{\prime}\right)=\left[f(x) / f\left(x_{0}\right)\right] p^{-1} .
$$

Hence $g \in M\left(\mu, J_{p}\right)$ satisf is $\alpha^{t} C_{0}=g$, and $g(x)=f(x) / f\left(x_{0}\right)$ for all $x \in \mu$. It follows that $\left(\alpha^{t}\right)^{-1}$ sends $f(x)$ onto $f\left(x_{0}\right) C_{0}$.

4.12. Proposition. $M_{b}\left(\mu, F_{p}\right)$ is homogeneous.

Proof. Let $x_{0}$ be an arbitrarily chosen point of $\mu$. Given a nonzero $g \in M_{b}\left(\mu, F_{p}\right)$, we show that $g$ may be sent to $C_{0}$ by a homeomorphic automorphism of $M_{b}\left(\mu, F_{p}\right)$. Permutations of $\mu$ give rise to homeomorphic automorphisms of $M_{b}\left(\mu, F_{p}\right)$, and homeomorphic automorphisms of $M\left(\mu, J_{p}\right)$ extend uniquely to homeomorphic automorphisms of $M_{b}\left(\mu, F_{p}\right)$, since $M_{b}\left(\mu, F_{p}\right)$ is a torsion-free divisible group, and $M\left(\mu, J_{p}\right)$ is an open subgroup. (See [4, Theorem A.7, p. 441].) Choose $n$ such that $p^{n} g=f$ is an element of $M\left(\mu, J_{p}\right)$. Then norm $f(x)$ attains a maximum at some point of $\mu$. Applying a permutation, we may as well assume that norm $f(x)$ attains a maximum at $x_{0}$. Multiplication by a unit of $J_{p}$ is a homeomorphic automorphism of $J_{p}$ which leaves invariant every subgroup contained in a basis at $0 \in J_{p}$. Hence we may define an automorphism of $M\left(\mu, J_{p}\right)$ which consists of pointwise multiplication by an element $h \in M\left(\mu, J_{p}\right)$ such that $h(x)$ is a unit for every $x \in \mu$. Every element of $J_{p}$ is $p^{n} \cdot u$, where $u$ is a unit. Thus pointwise multiplication by the appropriate $h(x)$ sends $f$ onto $h \cdot f$, where $[h \cdot f](x)=p^{n(x)}$ for every $x \in \mu$. Hence we may now assume that $f$ satisfies the hypotheses of Lemma 4.11. Applying Lemma 4.11 , we may as well assume that $f=p^{m} C_{0}$, and the proof will be complete once we show that there is a homeomorphic automorphism of $M_{b}\left(\mu, F_{p}\right)$ which sends $p^{m} C_{0}$ onto $C_{0}$. Here we must pass from properties of $J_{p}$ to properties of $F_{p}$, and use the fact that $F_{p}$ is a locally compact field with continuous field operations. Writing $\mu=x_{0}+\lambda$, we denote by $p^{-m} \oplus 1$ that automorphism of $M_{b}\left(\mu, F_{p}\right)=F_{p}+M_{b}\left(\lambda, F_{p}\right)$ which is defined by

$$
\left(p^{-m} \oplus 1\right)\left(t C_{0}\right)=p^{-m} t C_{0} \quad \text { and } \quad\left(p^{-m} \oplus 1\right)(g)=g \quad \text { if } \quad g\left(x_{0}\right)=0 .
$$

Then $p^{-m} \oplus 1$ sends $p^{m} C_{0}$ onto $C_{0}$, and we may combine the various homeomorphic automorphisms discussed in this proof to produce a homeomorphic automorphism which sends $g$ onto $C_{0}$. (Counterexample 2.4 shows the desirability of producing an element which is supported on a finite set.)

4.13. TheOREM. $A$ is a homogeneous group if and only if $A$ belongs to one of the four classes of groups $R^{n}, Z_{p}(\lambda, \mu), Q_{d}(\mu), M_{b}\left(\mu, F_{p}\right)$. 
Proof. The argument in Counterexample 4.3 shows that $Q_{d}(\mu)$ is homogeneous, and the same argument works for $R^{n} . Z_{p}(\lambda, \mu)$ and $M_{b}\left(\mu, F_{p}\right)$ are homogeneous by Propositions 4.9 and 4.12. The converse is a combination of Theorem 4.5, Proposition 4.7, and Counterexample 4.3, once the argument of 4.3 is extended in the obvious fashion from $\hat{Q}_{d}$ to $M\left(\mu, \hat{Q}_{d}\right)$.

4.14. Corollary. $(A, \hat{A})$ is a homogeneous dual pair if and only if $A$ belongs to one of the three classes $R^{n}, Z_{p}(\lambda, \mu), F_{p}^{n}$.

Proof. The proof is immediate, since 4.13 shows us explicitly which groups $A$ have the property that both $A$ and $\hat{A}$ are homogeneous.

5. Applications. We begin the applications of $\S 4$ by showing that $G$ is torsionfree if and only if $\hat{G}$ has a divisible dense subgroup.

5.1. Lemma. Suppose $G$ is a local direct product $L\left(X, G_{x}, K\left(G_{x}\right)\right)$ such that $X$ is a set of prime numbers, and $\left(G_{x}, K\left(G_{x}\right)\right)$ is, for each $x \in X$, the dual of some Z-pair $\left(M_{b}\left(\mu, F_{p}\right), M\left(\mu, J_{p}\right)\right)$. Then $G$ has a dense subgroup which is divisible.

Proof. If $x=p$, then $\left(G_{x}, K\left(G_{x}\right)\right)$ is the $Z$-pair $\left(M_{b}\left(\mu, F_{p}\right), M\left(\mu, J_{p}\right)^{\perp}\right)$, and so we will first show that the dual of $M_{b}\left(\mu, F_{p}\right)$ has a divisible dense subgroup. Let $D_{p}$ be the maximal divisible subgroup of $M_{b}\left(\mu, F_{p}\right)$. In order to show that $D_{p}$ is not the identity subgroup, we note that $M_{b}\left(\mu, F_{p}\right)$ has $F_{p}$ as a direct summand, and therefore $M_{b} \hat{b}\left(\mu, F_{p}\right)$ has $F_{p}$ as a direct summand, since $\hat{F}_{p}=F_{p}$. The divisibility of $F_{p}$ then implies that $D_{p}$ has more than one element, and so $D_{p}$ is dense in $M_{b}^{\wedge}\left(\mu, F_{p}\right)$, by 4.12 and 4.2. This establishes our lemma for the case when $X$ is a set consisting of just one prime number. For the general case, we make use of the fact that $L\left(X, G_{x}, K\left(G_{x}\right)\right)$ is the union of the groups $M\left(N, G_{x}\right)+M\left(X-N, K\left(G_{x}\right)\right)$, where $N$ is a finite subset of $X$. Let $D$ be the maximal divisible subgroup of $L\left(X, G_{x}, K\left(G_{x}\right)\right)$, and let $\bar{D}$ denote the closure of $D$. Then $\bar{D}$ contains every $M\left(N, K\left(G_{x}\right)\right)$ with $N$ finite, since $\bar{D}$ contains every $G_{x}=M_{b}\left(\mu, F_{x}\right)$ by the above. Thus $\bar{D}$ contains the entire compact open subgroup $M\left(X, K\left(G_{x}\right)\right)$, since the union of all $M\left(N, K\left(G_{x}\right)\right)$ forms a dense subgroup of $M\left(X, K\left(G_{x}\right)\right)$. It follows that $\bar{D}$ is all of $L\left(X, G_{x}, K\left(G_{x}\right)\right)$, since $\bar{D}$ contains $M\left(N, G_{x}\right)+M\left(X-N, K\left(G_{x}\right)\right)$. Thus $D$ is dense in $L\left(X, G_{x}, K\left(G_{x}\right)\right)$.

\subsection{THEOREM. $G$ is torsion-free if and only if $\hat{G}$ has a divisible dense subgroup.}

Proof. If $\hat{G}$ has a divisible dense subgroup, then $e \in G$ is the only character on $\hat{G}$ which has finite order, since the range of any other character must be an infinite subset of the circle. Thus $G$ is torsion-free. Conversely, suppose that $G$ is torsionfree. Using the theorem of Mackey which is quoted in the proof of Theorem 3.26, we see that $G$ is an open subgroup of $R^{n}+A+B+C$, where $A, B$, and $C$ are as in 3.26. Thus $\hat{G}$ is a quotient group of $R^{n}+\hat{A}+\hat{B}+\hat{C}$, where $\hat{A}=Q_{d}(\mu)$, and $\hat{B}$ $=M\left(\lambda, \hat{Q}_{d}\right)$. All of the groups $R^{n}, \hat{A}$, and $\hat{B}$ are divisible, and Lemma 5.1 shows that $\hat{C}$ has a divisible dense subgroup. (See [2, Theorem 1, p. 10].) Thus $\hat{G}$ has 
a divisible dense subgroup, since $\hat{G}$ is a quotient group of a group which has a divisible dense subgroup.

We now turn to the study of division rings. Assuming certain mild continuity restrictions, it follows that the additive group is very closely related to the additive group of a locally compact field. A complete classification of nondiscrete locally compact fields is given in [9, p. 189].

5.3. Definition. $D$ is a weakly continuous division ring if $D$ is algebraically a division ring, and the additive group of $D$ is a locally compact group such that each left multiplication operator is a homeomorphic automorphism.

5.4. Proposition. Let $G$ be the additive group of a weakly continuous division ring $D$. Then $G$ is isomorphic to one of the groups $R^{n}, Z_{p}(\lambda, \mu), Q_{d}(\mu), F_{p}^{n}$.

Proof. Theorem 4.13 shows that $G$ is one of the groups $R^{n}, Z_{p}(\lambda, \mu), Q_{d}(\mu)$, $M_{b}\left(\mu, F_{p}\right)$. Suppose $G$ is torsion-free. Given a character $\alpha \in \hat{G}$, define the homomorphism $h_{\alpha}: G \rightarrow \hat{G}$ by $h_{\alpha}(x)=\alpha \circ M_{x}$, where $M_{x}$ is the left multiplication operator $M_{x}(y)=x y$. Since $\alpha$ is in the range of the homomorphism $h_{\alpha}$, we see that every $\alpha \in \hat{G}$ is contained in a divisible subgroup of $\hat{G}$. Thus $\hat{G}$ is divisible, and we see by 2.7 that the groups $F_{p}^{n}$ are the only groups of the type $M_{b}\left(\mu, F_{p}\right)$ which can occur.

5.5. COROLlaRY. If $D$ is nondiscrete and has characteristic zero, then $G$ is either $R^{n}$ or $F_{p}^{n}$.

Proof. The proof is immediate.

5.6. Proposition. Suppose $D$ is a weakly continuous division ring such that multiplication is jointly continuous in both variables simultaneously. Then the additive group $G$ cannot be compact unless $D$ is finite.

Proof. Regarding $G$ as a set of left multiplication operators, it can be shown that every subset of $G$ which is open relative to the compact open topology is also open relative to the topology of $G$. Assuming $G$ compact, it follows that the set which maps $G$ onto some proper open subset is an open subset of $G$. This open subset must be the zero element, and hence $G$ is discrete.

REMARK. If $D$ satisfies the hypotheses of 5.6 and is nondiscrete, then it can be shown that the additive group $G$ is second countable and is isomorphic to $\hat{G}$. It follows that inversion is automatically continuous on $D-\{0\}$, so the multiplicative group of $D$ is a topological group. Moreover, the above can be taken as a starting point to show that $G$ is isomorphic to one of the groups $R^{n}, Z_{p}(\omega, \omega), Q_{d}(\mu), F_{p}^{n}$.

We now turn to the study of Aut $[G]$, the group of homeomorphic automorphisms of $G$. The word automorphism will always be understood to mean homeomorphic automorphism.

5.7. Proposition. The automorphism group of $M_{b}\left(\mu, F_{p}\right)$ acts transitively on the set of compact open subgroups of $M_{b}\left(\mu, F_{p}\right)$. 
Proof. First we show that all compact open subgroups of $M\left(\lambda, J_{p}\right)$ are isomorphic to $M\left(\lambda, J_{p}\right)$. Let $N$ be a finite subset of $\lambda$, and let $a_{x}, x \in N$ be an independent subset of $Z_{p}^{\infty}(\lambda)$. Choose the constants $c_{x}$ such that each $c_{x} a_{x}$ has order exactly equal to $p$. Map each $c_{x} a_{x}$ onto $p^{-1} C_{x}^{\prime}$ (Definition 4.10), and extend this mapping to a bijective automorphism $\tilde{h}$ of the vector space $A$, where $A$ is the subgroup of $Z_{p}^{\infty}(\lambda)$ consisting of all elements which have order at most $p$. Next, extend $\tilde{h}$ to an endomorphism $h$ from $Z_{p}^{\infty}(\lambda)$ to $Z_{p}^{\infty}(\lambda)$ [4, Theorem A.7, p. 441]. Then $h$ must be a bijective automorphism, since $h$ is bijective on $A$, and $Z_{p}^{\infty}(\lambda)$ is the minimal divisible extension of $A$. The subgroup generated by the $a_{x}$ is mapped by $h$ onto a subgroup $B$, where $B$ is a finite subgroup of $Z_{p}^{\infty}(\lambda)$ which is supported on the finite set $N$, and is generated by the elements $p^{-n(x)} C_{x}^{\prime}$. Using the fact that all the finite subgroups of $Z_{p}^{\infty}$ have isomorphic quotient groups, it now follows that all the finite subgroups of $Z_{p}^{\infty}(\lambda)$ have isomorphic quotient groups. Thus all the compact open subgroups of $M\left(\lambda, J_{p}\right)$ are isomorphic.

Turning to the case of $M_{b}\left(\mu, F_{p}\right)$, we recall that $M_{b}\left(\mu, F_{p}\right)$ is a torsion-free topological torsion group which is the minimal divisible extension of any compact open subgroup (2.5). Thus it suffices to establish isomorphisms between compact open subgroups, since these isomorphisms can always be extended (uniquely) to homeomorphic automorphisms of $M_{b}\left(\mu, F_{p}\right)$. Let $K_{1}$ be an arbitrary compact open subgroup. If $K_{2}=K_{1}+M\left(\mu, J_{p}\right)$ were isomorphic to $M\left(\mu, J_{p}\right)$, then $K_{1}$ would be isomorphic to a compact open subgroup of $M\left(\mu, J_{p}\right)$, and hence isomorphic to $M\left(\mu, J_{p}\right)$ by the above. Thus it suffices to show that $K$ is isomorphic to $M\left(\mu, J_{p}\right)$ when $K$ contains $M\left(\mu, J_{p}\right)$. The quotient group $K \bmod M\left(\mu, J_{p}\right)$ has $p^{n}$ elements, and induction on $n$ reduces the problem to the case $n=1$. Let $f$ be an element of $M\left(\mu, J_{p}\right)$ satisfying $p^{-1} f \notin M\left(\mu, J_{p}\right)$, and let $K=p^{-1} f+M\left(\mu, J_{p}\right)$. Then the proofs of 4.11 and 4.12 show that $K$ is isomorphic to $p^{-1} C_{0}+M\left(\mu, J_{p}\right)$. Thus $K$ is isomorphic to $M\left(\mu, J_{p}\right)$, since $p J_{p}$ is isomorphic to $J_{p}$.

5.8. Definitions. Let Aut $[G]$ denote the group of all homeomorphic automorphisms of $G$. We equip Aut $[G]$ with the topology described in $[4$, p. 426], a topology which yields a (not necessarily locally compact) topological group. Suppose $G$ is totally disconnected and $F$ and $H$ are compact open subgroups with $H \subset F$. The topology in question has as a typical (open) basis neighborhood of the identity the set $\Gamma(F, H)$, namely, those automorphisms which map every coset of $F / H$ onto itself (see [4, Theorem 20.8, p. 428]). An automorphism $\alpha$ is in $\Gamma(F, H)$ if $\alpha(x+H)=x+H$ whenever $x \in F$. (It can be shown that this topology for Aut $[G]$ coincides with the $g$-topology defined in [1, p. 595].) In general, we will use the notation $\Gamma(F, H)$ with the meaning above whenever $F$ and $H$ are closed subgroups.

5.9. Lemma. Suppose $G$ has an open subgroup $A$ such that every element of Aut $[A]$ can be extended to an element of $\Gamma_{G}(A, A)$. Then $\Gamma_{G}(A, A)$ is closed in Aut $[G]$, and the restriction mapping from $\Gamma_{G}(A, A)$ to Aut $[A]$ is a continuous surjective homomorphism. 
Proof. Given any point $x_{0}$ in $G$, the set of automorphisms which map $x_{0}$ into a neighborhood of itself is an open neighborhood of the identity in Aut [G], and it follows that $h_{\alpha}\left(x_{0}\right) \rightarrow h\left(x_{0}\right)$ whenever $h_{\alpha} \rightarrow h$ in Aut [G]. Thus the assumption that $A$ is open (hence closed) is easily seen to imply that $\Gamma_{G}(A, A)$ is closed in Aut $[G]$. In order to show that the restriction mapping from $\Gamma_{G}(A, A)$ to Aut $[A]$ is continuous, choose $\Gamma_{A}(F, H)$ a typical basic neighborhood of the identity in Aut $[A]$. Here $F$ and $H$ are compact open subgroups of $A$, and hence also of $G$, since $A$ is open. Thus the assumption that $A$ is open implies that $\Gamma_{G}(F, H)$ is open in Aut $[G]$, and so the restriction mapping is continuous, since $\Gamma_{G}(F, H) \cap \Gamma_{G}(A, A)$ is by definition open in $\Gamma_{G}(A, A)$.

5.10. Lemma. Let $G=M_{b}\left(\mu, F_{p}\right)$. If Aut $[G]$ were locally compact, Aut $[K]$ would be locally compact, where $K=M\left(\mu, J_{p}\right)$.

Proof. If Aut $[G]$ is locally compact, then there is a pair of compact open subgroups $F \supset H$ such that $\Gamma(F, H)$ is a compact subgroup of Aut [G]. Proposition 2.5 can be used to show that $F$ may be taken as the group $A$ of Lemma 5.9, since divisible groups are precisely the category where homomorphisms can always be extended. The restriction mapping of Lemma 5.9 maps $\Gamma_{G}(F, H)$ onto $\Gamma_{F}(F, H)$, and the latter is an open subgroup of Aut $[F]$ by definition. Thus Lemma 5.9 shows that Aut $[F]$ is locally compact, since $\Gamma_{G}(F, H)$ is a compact subgroup of $\Gamma_{G}(F, F)$. Using Proposition 5.7 we see that $F$ is isomorphic to $M\left(\mu, J_{p}\right)$, and Aut $\left[M\left(\mu, J_{p}\right)\right]$ is then locally compact.

5.11. Lemma. Let $\mu$ be infinite, and suppose that $G$ is either $Z_{p}^{\infty}(\mu)$ or else one of the discrete vector spaces $Q_{d}(\mu), Z_{p}(\mu)$. Then Aut $[G]$ fails to be locally compact.

Proof. We show that the proof of [4, section 26.18(j), p. 435] can be adapted to the case at hand. Suppose $G=Z_{p}^{\infty}(\mu)$, and suppose that Aut $[G]$ is locally compact. Then some subgroup $\Gamma(F, e)$ is compact, where $F$ is a finite subgroup consisting of the elements $a_{j}$. Each $a_{j}$ is a finite combination $\sum_{k} a_{j}(k) C_{k}^{\prime}$, where $a_{j}(k)=p^{-n(j, k)}$ (see Definition 4.10). Let $n$ be the largest $n(j, k)$ which appears in the expressions for elements of $F$. Define $E$ as the subgroup generated by the elements $p^{-n} C_{k}^{\prime}$, where every $C_{k}^{\prime}$ is included which appears in the expression for some $a_{j}$. Then $\Gamma(E, e)$ is a subgroup of $\Gamma(F, e)$, and so $\Gamma(E, e)$ is compact. Choose some $C_{m}^{\prime}$ such that $p^{-n} C_{m}^{\prime}$ is not in $E$, and cover $E$ by translates of the subgroup $B$, where $B$ consists of those elements which fix $p^{-n} C_{m}^{\prime}$ and also leave $E$ pointwise invariant. Then the proof quoted above shows that the automorphisms which fix $E$ pointwise can only map the element $p^{-n} C_{m}$ a finite number of places. This contradicts what Proposition 5.7 tells us about the finite subgroups of $Z_{p}^{\infty}(\mu)$, since isomorphisms between finite subgroups can be extended to automorphisms of $Z_{p}^{\infty}(\mu)$. This finishes the proof when $G=Z_{p}^{\infty}(\mu)$, and the proof for infinite dimensional vector spaces follows readily from the arguments above.

5.12. TheOREM. Let $G$ be a homogeneous group. Then Aut $[G]$ is locally compact if and only if $G$ is one of the finite dimensional vector spaces $R^{n}, F_{p}^{n}, Q_{d}^{n}$, or $Z_{p}^{n}$. 
Proof. All of the groups listed in the conclusion of the theorem have the property that a continuous endomorphism is necessarily a linear map. Thus Aut $[G]$ is a matrix group, and is locally compact by the usual argument of continuity of the determinant. If $G$ is a homogeneous group, then Theorem 4.13 shows that $G$ is one of the groups $R^{n}, Z_{p}(\lambda, \mu), Q_{d}(\mu), M_{b}\left(\mu, F_{p}\right)$. Lemma 5.11 shows that Aut $\left[Q_{d}(\mu)\right]$ fails to be locally compact when $\mu$ is infinite. Let $G=M_{b}\left(\mu, F_{p}\right)$ with $\mu$ infinite. If Aut $[G]$ is locally compact, then $K=M\left(\mu, J_{p}\right)$ is locally compact, by Lemma 5.10. However, $[4$, Theorem 26.9 , p. 429$]$ shows that Aut $[A]$ and Aut $[\hat{A}]$ are always anti-isomorphic, and hence isomorphic, since inversion is an anti-automorphism. Thus Lemma 5.11 shows that $\mu$ cannot be infinite. Now let $G=Z_{p}(\lambda, \mu)$. The crucial steps in Lemmas 5.10 and 5.9 are the facts that compact open subgroups are all isomorphic, and that automorphisms of compact open subgroups can be extended to give automorphisms of the entire group. These statements are easily seen to be valid for $Z_{p}(\lambda, \mu)$, and so Aut $\left[M\left(\mu, Z_{p}\right)\right]$ would be locally compact if Aut $\left[Z_{p}(\lambda, \mu)\right]$ were locally compact. As above, this would be equivalent to the assertion that $\operatorname{Aut}\left[Z_{p}(\mu)\right]$ is locally compact, and Lemma 5.11 shows that $\mu$ cannot be infinite. $Z_{p}(\mu, \lambda)$ is the dual of $Z_{p}(\lambda, \mu)$, and so $\lambda$ must also be finite. Thus $Z_{p}(\lambda, \mu)=Z_{p}^{n}$, where $n=\lambda+\mu$.

5.13. Counterexample. Aut $[G]$ need not be locally compact, even if $G$ is the additive group of a nondiscrete locally compact field. Let the locally compact field have characteristic $p$. Then $G=Z_{p}(\omega, \omega)$, and automorphisms of the additive structure need not be automorphisms of whatever vector space structure the field may have, since $Z_{p}(\omega, \omega)$ does not appear in the list in Theorem 5.12. (The observation that $Z_{p}(\omega, \omega)$ is isomorphic as a topological group to the local direct product $L\left(\omega, Z_{p}(\omega, \omega), M\left(\omega, Z_{p}\right)\right)$ might lead one to suspect that $A u t\left[Z_{p}(\omega, \omega)\right]$ is rather too big to be locally compact.)

\section{Definitions AND Notation}

$\begin{array}{lclr}A_{p} & 3.19 & M\left(\mu, \hat{Q}_{d}\right) & 2.8 \\ \text { Aut }[G] & 5.8 & M\left(\mu, Z_{p}\right) & 2.11 \\ C_{0}, C_{x}, p^{-n} C_{x}^{\prime} & 4.10 & \text { norm } & 4.10 \\ E(G) & 1.0 & p \text {-summand } & 3.19 \\ \text { endomorphism-simple } & 4.1 & p^{-n} C_{x}^{\prime} & 4.10 \\ F_{p} & 2.1 & Q_{d}, \hat{Q}_{d}, Q_{d}(\mu) & 2.8 \\ \Gamma(F, H) & 5.8 & R & \text { the real line } \\ \text { homogeneous } & 4.1 & T & 2.1 \\ J_{p} & 2.1 & \text { topological } p \text {-group } & 3.1 \\ \lambda & \text { a cardinal number } & \text { topological torsion } & 3.1 \\ L\left(\mu, G_{x}, K\left(G_{x}\right)\right) & 1.0 & \omega & \text { the first inf. card. } \\ \text { local direct product } & 1.0 & \text { wkly. cont. div. ring } & 5.3 \\ \text { locally quasi-connected } & 3.4 & Z \text {-groups, } Z \text {-pairs } & 1.0 \\ \mu & \text { a cardinal number } & Z_{p}, Z_{p}(\mu), Z_{p}(\lambda, \mu) & 2.11 \\ M\left(\mu, J_{p}\right), M_{b}\left(\mu, F_{p}\right) & 2.1 & Z_{p}^{\infty}, Z_{p}^{\infty}(\mu) & 2.1\end{array}$




\section{BIBLIOGRAPHY}

1. R. Arens, Topologies for homeomorphism groups, Amer. J. Math. 68 (1946), 593-610.

2. J. Braconnier, Sur les groupes topologique localement compacts, J. Math. Pures Appl. 27 (1948), 1-85.

3. E. Enochs, Homotopy groups of compact abelian groups, Proc. Amer. Math. Soc. 15 (1964), 878-881.

4. E. Hewitt and K. Ross, Abstract harmonic analysis. I, Academic Press, New York, 1963.

5. I. Kaplansky, Infinite Abelian groups, Univ. of Michigan Press, Ann Arbor, Mich., 1954.

6. A. Kurosh, Lectures on general algebra, Chelsea, New York, 1963.

7. G. W. Mackey, A remark on locally compact abelian groups, Bull. Amer. Math. Soc. 52 (1946), 940-944.

8. L. S. Pontrjagin, Topological groups, Princeton Univ. Press, Princeton, N. J., 1939.

9. — Topologischen Gruppen. I, II, 2nd ed., Teubner, Leipzig, 1957-1958.

10. A. Weil, L'integration dans les groupes topologique et ses applications, Actualitiés Sci. Ind. Nos. 869, 1145, Herman, Paris, 1941.

\section{UNIVERSITY OF WASHINGTON,}

SeATtLe, Washington 